\title{
B Vitamins for Neuropathy and Neuropathic Pain
}

Mauro Geller ${ }^{1}$, Lisa Oliveira ${ }^{1}$, Rafael Nigri ${ }^{1}$, Spyros GE Mezitis ${ }^{2}$, Marcia Gonçalves Ribeiro ${ }^{3}$, Adenilson de Souza da Fonseca ${ }^{4}$, Oscar Roberto Guimarães ${ }^{5}$, Renato Kaufman ${ }^{6}$ and Fernanda Wajnsztajn 6

${ }^{1}$ Federal University of Rio de Janeiro, Rio de Janeiro, Brazil

${ }^{2}$ New York-Presbyterian Hospital/Weill-Cornell Medical Center, New York, USA

${ }^{3}$ Federal University of Rio de Janeiro, Rio de Janeiro, Brazil

${ }^{4}$ UNIFESO Medical School, Teresópolis, Brazil

${ }^{5}$ State University of Rio de Janeiro, Rio de Janeiro, Brazil

${ }^{6}$ New York-Presbyterian Hospital/ Columbia University Medical Center, New York, USA

*Corresponding author: Mauro Geller, Federal University of Rio de Janeiro, Brazil Av. Ataulfo de Paiva, 135 sl. 1104, Brazil, Tel: (+55-21)3875-6660; Fax: (+55-21)2259-3395; E-mail: maurogeller@gmail.com

Received date: June 01, 2017; Accepted date: June 21, 2017; Published date: June 28, 2017

Copyright: (c) 2017 Geller M, et al. This is an open-access article distributed under the terms of the Creative Commons Attribution License, which permits unrestricted use, distribution, and reproduction in any medium, provided the original author and source are credited.

\section{Abstract}

Vitamins B1, B6 and B12 are members of a group of water-soluble organic vitamins with important structural and functional roles in the human body. This updated literature review examines the physiological and biochemical properties of these B vitamins.

Keywords: Vitamin B1; Vitamin B6; Vitamin B12

\section{Introduction}

The B vitamins are a group of water-soluble organic compounds which, while structurally diverse, all play crucial roles in the maintenance of body functions, including proper cellular function, tissue growth and development $[1,2]$. The B vitamin family includes vitamin B1 (thiamine), B2 (riboflavin), B3 (niacin), B5 (pantothenic acid), B6 (pyridoxine), B9 (folate), and B12 (cobalamin). With the exception of vitamin B3, which can be synthesized from tryptophan, all of the B vitamins the human body requires must be provided from a dietary or exogenous source. Consequently, B-vitamin deficiency may arise within weeks of inadequate intake, depending on the $\mathrm{B}$ vitamin. The B complex vitamins (B1, B6 and B12) play fundamental roles in the nervous system both structurally and in maintenance of proper nervous system functions. Increasingly there is evidence in the literature to suggest that the $\mathrm{B}$ complex vitamins contribute to promoting nerve repair, both in acceleration of nerve tissue regeneration and recovery of nerve function by a variety of mechanisms $[3,4]$.

\section{Neuropathic, nociceptive and mixed pain}

In any discussion of the participation of $B$ vitamins in neuropathy and neuropathic pain, it is important to briefly revisit the definitions and the different pain types. The International Association for the Study of Pain defines pain as "an unpleasant sensory and emotional experience associated with actual or potential tissue damage or described in terms of such damage" [5]. Pain classifications vary and may include intensity, duration, and underlying mechanisms. In terms of underlying mechanisms, pain can be divided into two broad categories: nociceptive pain and neuropathic pain.

Neuropathic pain is defined as "pain arising as a direct consequence of a lesion or disease affecting the somatosensory system" [6] and can be classified based on its origin as central or peripheral, and also by etiology, signs and symptoms, and mechanisms. Neuropathic pain may arise from many different conditions affecting the central and/or peripheral nervous system, occurring at any point along nerve pathways from the peripheral nerves to the cortical neurons, although the precise underlying mechanisms are not yet fully understood and vary among individuals. It is a chronic condition and is associated with negative impact on quality of life, increased disability, and increased costs [7]. Examples of prevalent neuropathic pain conditions include diabetic, alcoholic, and chemotherapy-induced polyneuropathy, nerve compression or infiltration by tumor, post-herpetic neuralgia, complex regional pain syndrome, and inflammatory demyelinating polyradiculoneuropathy [8].

In contrast to neuropathic pain, nociceptive pain has a protective function, occurring as a consequence of nociceptor activation in response to actual or potential damage to non-neural tissue. Noxious stimuli triggering nociceptive pain may be external or internal and are generally categorized as thermal, mechanical, and chemical. Nociceptive pain may be further subcategorized as somatic (manifesting in musculoskeletal or cutaneous regions) or visceral (affecting hollow organs and smooth muscle) [9]. It is also important to note the difference between nociception and pain. Pain perception occurs in the cerebral cortex, the final destination for noxious stimuli which activate nociceptors in peripheral structures, which in turn transmit the information to the dorsal horn or nucleus caudal is, ascending to the brainstem and finally the cerebral cortex.

Mixed pain is a fairly recent concept, encompassing chronic pain conditions including low back pain, cancer pain, and osteoarthritis, presenting characteristics of both nociceptive and neuropathic pain types. Mixed pain represents a challenge for physicians and requires careful evaluation of the patient and multimodal, individualized treatment approaches [10]. 


\section{Discussion}

\section{Vitamin B1: Dietary sources, functions and deficiency}

Vitamin B1 or thiamine can be obtained from foods such as whole grains, dairy products, and red meat. The recommended dietary allowance (RDA) for vitamin B1 is $1.2 \mathrm{mg} /$ day and $1.1 \mathrm{mg} /$ day for men and women, respectively [11]. Thiamine acts as both a biochemical coenzyme precursor in metabolic functions and as a structural component in biological membranes of the peripheral and central nervous system. The physiologically active form of thiamine is thiamine pyrophosphate (or thiamine diphosphate), where it functions as an essential enzyme for carbohydrate metabolism and in the brain, it plays a key role in glucose and energy metabolism [12].

Examples of participation of thiamine in metabolic processes include its use as a co-enzyme in reactions catalyzing the transfer of two-carbon units, such as dehydrogenation of 2-oxoacids: 2oxoglutarate dehydrogenase, trans ketolase, and pyruvate dehydrogenase - all of which are important in carbohydrate metabolism [13]. Thiamine pyrophosphate is a co-factor in the three enzymatic systems (the mitochondrial pyruvate dehydrogenase complex, the a-ketoglutarate dehydrogenase complex, and the cytosolic trans ketolase) responsible for cerebral glucose metabolism [12]. Thiamine also functions as a catalyst in conjunction with coenzymes such as flavin and NAD, in the conversion of pyruvate to acetyl $\mathrm{CoA}$, an oxidative decarboxylation reaction mediated by pyruvate dehydrogenase [13].

Thiamine has been identified as an active cellular membrane component in axoplasms, synaptosomes, and mitochondria, and plays an important role in maintenance of cell membrane stability, exerting physiologically protective actions on nervous cells, especially axonal membranes and other tissues [14]. During embryogenesis, thiamine stabilizes the membranes of newly generated neuronal cells, slows developmental apoptosis, and contributes to plasma membrane transformation of uterine epithelial cells [14]. Thiamine is also important in the electrical function of neuronal membranes. During synaptic transmission, thiamine undergoes both axonal anterograde and retrograde transmission, and has been proposed to play a role in regulation of membrane permeability to sodium during the action potential and maintenance of electronegativity at the inner surface membrane [15]. Experimentally, thiamine exerts an acute excitatory effect on hippocampal neuron membrane ion channels, with a significant increase in the number of repetitive after discharges, likely involving potassium channel [16]. Phosphorylated thiamine functions as an endogenous potassium ion $(\mathrm{K}+)$ channel blocker in neuronal cells [17]. Thiamine has been shown to exert a protective effect of the cellular membrane against ethanol-induced cytotoxicity, and to exert a nonspecific stabilizing effect on the axonal membrane [18].

Thiamine absorption occurs in the gastrointestinal (GI) tract and although it is widely distributed throughout the body, it is not stored at appreciable levels [19]. Thiamine deficiency may arise within 2 to 3 weeks of inadequate intake, be it from poor nutrition, alcohol abuse, illness, or surgical procedures, and primarily affects the nervous system, the gastrointestinal tract, and the cardiovascular system [20]. In cerebellar granule neurons, thiamine deficiency results in suppression of A-type $\mathrm{K}+$ channel cells, consequently provoking a significant decrease in voltage-dependent $\mathrm{K}+$ membrane conductance [21]. Other deficiency-associated nervous system alterations include reduction in nervous conduction speed and disturbances in nervous electrical activities arising from alterations to myelinogenesis, which consequently results in a diameter reduction of myelinic fibers [18].

Clinically, long-term thiamine deficiency is associated with a distal, sensorimotor polyneuropathy, especially in the lower limbs [22]. Polyneuropathy due to thiamine deficiency has been described as slow, bilateral, distal, painful paresthesia's often referred to as burning feet. Untreated, this progresses to gait alterations (steppage gait) and lower limb muscle weakness [22]. Wernicke's encephalopathy is a common consequence of B1 deficiency, often with a clinical presentation of acute onset nystagmus and ophthalmoplegia, changes in mental status, and unstable stance and gait [20]. Other early clinical signs include bilateral vision loss, hearing loss, hallucinations, stupor, tachycardia, hypotension, and behavioral alterations. Left untreated, Wernicke's encephalopathy may progress to complete ophthalmoplegia, spastic paresis and increased muscular tone, hyperthermia, choric dyskinesisas, and coma [20]. The chronic form of Wernicke's encephalopathy is Korsakoff's syndrome, arising from structural brain lesions. The clinical picture includes emotional alterations, learning impairment, and a noted loss of working memory while reference memory is relatively unaffected [23]. Risk factors for Korsakoff's syndrome include the traditionally recognized alcohol abuse, as well as non-alcohol related disease caused by thiamine deficiency itself, a combination of the two, a genetic predisposition among individuals with trans ketolase variants with reduced affinity for thiamine pyrophosphate [24].

Treatment of thiamine deficiency via parenteral, oral, or intramuscular administration of high doses of thiamine is indicated to rapidly and fully replenish serum levels, although clinical guidelines vary on optimal dose and duration of treatment within the different deficiency states [25]. While thiamine administration completely reverses the cardiovascular and GI symptoms of B1 deficiency, recovery from the peripheral nervous system alterations varies depending on their duration and severity [20].

\section{Vitamin B6: Dietary sources, functions and deficiency}

Vitamin B6 is found naturally in many food sources such as fish, beef, starchy vegetables, and fruit, and is also produced by the intestinal microflora. The RDA of vitamin B6 for adults 19-50 years of age $1.3 \mathrm{mg} /$ day for both genders. For individuals over 50 years old, $\mathrm{RDA}$ is $1.7 \mathrm{mg} /$ day for males and $1.5 \mathrm{mg} /$ day for females; pregnant women regardless of age require $1.9 \mathrm{mg} / \mathrm{day}$, while during lactation the $\mathrm{RDA}$ increases to $2.0 \mathrm{mg}$ [11]. Of the seven known forms of vitamin B6, pyridoxine is the most commonly used in B6 supplement preparations. The metabolically active form of vitamin B6 is pyridoxal 5'-phosphate (PLP), which is converted from pyridoxine, pyridoxamine, and pyridoxal, three related pyrimidine derivatives [26].

Vitamin B6 possesses two biologically distinct functions, acting as a co-factor in many metabolic, physiologic, and developmental processes and also as an antioxidant [27]. PLP is a co-factor for several enzymes catalyzing more than 140 essential enzymatic reactions, including participation in the metabolism of glucose, amino acids, and fatty acids, synthesis of histamine, hemoglobin, and neurotransmitters, and gene expression. PLP-dependent enzymes catalyze transamination decarboxylation, racemization, and $\alpha, \beta$-elimination reactions in amino acid metabolism, while functioning as an essential co-factor for enzymes involved in the biosynthesis of sphingolipids in lipid metabolism [28,29]. Transformation reactions catalyzed by PLP provide the amino acid substrates required for glucose metabolism. The PLP-dependent enzyme glycogen phosphorylase, essential to 
glucose metabolism, acts as a proton donor or receptor, mediating glycogen breakdown and the release of glucose from glycogen [30].

In hemoglobin synthesis, Vitamin B6 acts as a co-factor for the enzyme $\delta$-aminolaevulinic acid synthase, leading to primary biosynthesis of $\delta$-aminolaevulinic acid [31], while also binding to two sites on hemoglobin which enhance oxygen binding [32]. PLP also acts as a co-factor for enzymes involved neurotransmitter synthesis, most notably epinephrine, norepinephrine, serotonin, and $\gamma$-aminobutyric acid (GABA). DOPA carboxylase is a PLP-dependent enzyme involved in both serotonin and dopamine biosynthesis. Serotonin synthesis from L-tryptophan requires both tryptophan hydroxylase and DOPA (L-dihydroxyphenyl alanine) decarboxylase, in order to catalyze the conversion from 5-hydroxy-tryptophan to serotonin [33]. The initial precursor in dopamine synthesis is L-tyrosine, which is converted to LDOPA by L-tyrosine hydroxylase. In turn, L-DOPA is converted to dopamine in a reaction mediated by DOPA decarboxylase [32]. The PLP-dependent enzyme L-glutamate decarboxylase catalyzes the decarboxylation reaction from L-glutamate required in GABA synthesis [34]. The neuromodulator D-serine relies on action of the PLP-dependent enzyme serine racemase for its synthesis [35].

The antioxidant role of Vitamin B6 was first reported in 1999, when Ehrenshaft et al. reported singlet oxygen quenching by pyridoxine at a rate comparable to that of vitamin $\mathrm{C}$ and vitamin $\mathrm{E}$ [36]. Later studies confirmed the antioxidant properties of vitamin B6, including prevention of hydrogen peroxide-induced oxygen radical formation and lipid peroxidation in U937 monocytes [37]. Reduced levels of vitamin $\mathrm{B} 6$ have been associated with an increased susceptibility to abiotic stress, including oxidation, salinity, drought, and UV-B radiation in different organisms [38].

Following oral ingestion, pyridoxine is rapidly absorbed from the healthy GI tract. Factors that may contribute to Vitamin B6 deficiency include malnourishment, malabsorption, alcohol abuse, and some medications. Alcohol abuse leads to production of acetaldehyde, which competes with PLP for binding sites of PLP-dependent enzymes [25]. Drugs such as valproate, carbamazepine, and phenytoin increase Vitamin B6 breakdown, while other such as isoniazid and hydrocortisone interfere with its absorption. Other conditions identified as risk factors for B6 deficiency include HIV infection, sickle cell disease, hyperoxaluria, inflammation and tissue injury, and hereditary sideroblastic anemia, among others [39].

Vitamin B6 deficiency is associated with accumulation and urinary excretion of xanthurenic acid, an intermediate tryptophan metabolite, together with a reduction of glutamic oxaloacetic transaminase activity in red blood cells. In general, the signs of B6 deficiency among adults are evidenced in the peripheral nervous system, the skin, mucous membranes, and the hematopoietic system, while children are more prone to display central nervous system effects of B6 deficiency. The symptoms of B6 deficiency, which include seborrheic dermatitis, microcytic anemia, depression and confusion, are completely reversed by pyridoxine supplementation [40]. Vitamin B6 deficiency has also been linked to seizures of several types (partial, myoclonic, and generalized tonic-clonic). Although rare, these seizures are thought to be related to imbalances in cerebral amine neurotransmitter production, and while unresponsive to anticonvulsant drugs, they do respond to pyridoxine supplementation [26]. Vitamin B6 deficiency in adults produces a length dependent neuropathy beginning at the feet and ascending the lower limbs; if left untreated it will eventually reach the upper limbs. The early signs of this deficiency neuropathy include burning pain or numbness and paresthesia's and on neurological examination decreased distal sensation and reduction of deep tendon reflexes are evident, often accompanied by ataxia and mild distal weakness [41]. Vitamin B6 deficiency may also be a causative factor in idiopathic carpal tunnel syndrome, and supplementation with the vitamin has been reported to alleviate symptoms [42]. Pyridoxine deficiency may be corrected with supplementation, especially in cases of drug-induced deficiency, or drug discontinuation [39].

\section{Vitamin B12: Dietary sources, functions and deficiency}

The term vitamin B12 is used to refer to the cobalamins, a group of cobalt-containing corrinoids. Dietary sources of hydroxocobalamin, the form present in food, include animal products such as fish, beef, poultry, and dairy products. Vitamin B12 is synthesized in some bacteria, but not in plants and animals, thus dietary intake is important to maintain adequate levels and prevent deficiency [43]. The RDA for vitamin B12 in adults is $2.4 \mu \mathrm{g} /$ day. As the largest and most chemically complex of all the vitamins B12 functions as an enzymatic co-factor in a wide variety of biological reactions [44]. The metabolically active forms of B12 are adenosyl cobalamin, methyl cobalamin, and hydroxocobalamin; while cyanocobalamin, present in supplements, has no known direct biological role and must be converted into either adenosyl cobalamin or methyl cobalamin [43-45]. Vitamin B12 is required in the human body for the maintenance of normal erythropoiesis, cell reproduction and growth, as well as synthesis of nucleoproteins and myelin. Although the precise mechanisms of B12mediated myelination have not yet been entirely elucidated, methyl cobalamin has been shown to promote the synthesis of lecithin, one of the principle ingredients of myelin sheath lipids [46].

The B12-dependent enzymes can be divided into those relying on methyl cobalamin as a cofactor and those that rely on adenosyl cobalamin. Methyl cobalamin is important in the metabolism of propionic acid and the conversion of $\alpha$-leucine to $\beta$-leucine, and acts as a co-factor for the enzymes methionine synthase and L-methyl malonyl-CoA mutase. Methionine synthase relies on vitamin B12 as a co-factor for the methyl transfer from methyl tetrahydrofolate to homocysteine in the synthesis of methionine and tetrahydrofolate [47]. Vitamin B12 is also involved in the production of S-adenosyl methionine from homocysteine, with S-adenosyl methionine acting as the methyl donor in the maintenance of methylation patterns in DNA that determine gene expression and DNA conformation [48]. Adenosyl cobalamin is an essential co-factor of several enzymes, including LMethyl malonyl-CoA mutase, in the isomerization reaction that converts L-methyl malonyl-CoA to succinyl-CoA, a key molecule in the citric acid cycle [43].

The regeneration of tetrahydrofolate from its inactive form of 5methyltetrahydrofolate requires B12 as a coenzyme, and its absence leads to a functional folate deficiency [15,44]. Vitamin B12's role in the remethylation of homocysteine to methionine for de novo synthesis of s-adenosyl methionine makes it important to nerve metabolism. In protein synthesis as well as fat and carbohydrate metabolism, B12 acts in the maintenance of sulfhydryl groups in the reduced form required by many sulfhydryl-activated enzyme systems $[15,43,44]$.

The release of cobalamin from food proteins requires the presence of an acidic environment in the stomach, thus elderly patients and those undergoing acid reduction therapy may be at risk for cobalamin deficiency [49]. Upon ingestion, gastrointestinal absorption of vitamin B12 relies on the presence of intrinsic factor, a gastric mucosa-secreted glycoprotein that binds B12 prior to absorption. The trans cobalamins, particularly trans cobalamin II, are a group of plasma proteins that 
bind vitamin B12 and act in the rapid transport of B12 to tissues [50] Ilea absorption of B12 may be affected by several different conditions: in patients lacking intrinsic factor, those presenting a malabsorption syndrome, dietary habits (vegetarianism/veganism), or as a result of alteration to the GI tract due to disease, anatomic abnormality or following gastrectomy.

The underlying causes of Vitamin B12 deficiency are often inadequate intake, poor absorption, or a combination of the two. The principal clinical manifestations of $\mathrm{B} 12$ deficiency include megaloblastic anemia, gastrointestinal lesions and neurological damage due to the disruption of myelin formation followed by gradual degeneration of the axon and nerve head, the clinical signs of which are myelopathy and neuropathy [51]. The neurological symptoms of B12 deficiency have been attributed to the absence of s-adenosyl methionine formation, disrupting reactions beginning with this compound. Another important aspect of B12 deficiency is the resultant elevation of total plasma homocysteine, increasing risk of thrombosis and stroke [52]. B12 supplementation leads to complete reversal of megaloblastic anemia and gastrointestinal alterations associated with B12 deficiency. Supplementation also halts progression of neurological lesions, although resolution of neurological symptoms may take up six months or more, depending on the duration of the deficiency and the severity of the lesions $[39,43]$.

\section{Multiple B vitamin deficiency: Interactions and Implications of Poly-Deficiency}

It is important to note that the B vitamins often work in synergy with one another in biochemical and physiological processes [25]. Thus, the concept of poly-deficiency must be taken into account when assessing and treating a given B vitamin deficiency, as there may be a concomitant unidentified deficiency of another of the B vitamins. One clear example of this situation is in folate (vitamin B9) deficiency, in which thiamine absorption is impaired as a result of frequent diarrhea arising from damage to the gastrointestinal tract caused by the folate deficiency. This damage in turn hampers proper absorption of other vitamins and nutrients, including folate itself [53]. Folate deficiency at the cellular level and its resultant decline in dihydrofolate reductase activity can also interfere with thiamine activation, indirectly contributing to thiamine deficiency even in the presence of sufficient thiamine [54]. Thiamine, in turn, may impact pyridoxine metabolism in situations of altered B1 metabolism [55].

Specific situations - such as alcoholism - are favorable to the development of multiple B vitamin deficiencies, with B1 deficiency reported among $30-80 \%$ of alcoholics, B6 deficiency in 50\%, and B12 deficiency in $17 \%$ [56]. Among adults over 65 years of age, a subdeficiency of both vitamin B2 and vitamin B6 has been noted, and supplementation among this population with riboflavin results in a return to normal levels not only of riboflavin itself but also of pyridoxine [39]. Metabolically, several of the B vitamins rely on the biologically active forms of riboflavin in order to properly function, and consequently a B2 deficiency could give rise to deficiencies in folate, vitamin B6, and vitamin B12. For instance, cobalamin synthesis requires the presence of flavin adenine dinucleotide [57]. Another example of this dependency is the conversion of pyridoxine to PLP, which relies on pyridoxine 5 '-phosphate oxidase, a flavin mononucleotide-dependent enzyme [58]. While results have varied especially among different age brackets, the relationship between folate and B12 status has been noted among senior populations, with an increased risk of cognitive impairment and anemia upon exposure to high levels of folate in individuals with sub-optimal B12 status [59].

There is a great deal of interest surrounding a possible role of the B vitamins in protecting against neurodegeneration, particularly among the aging population. It is hoped that the $\mathrm{B}$ vitamins and other micronutrients and vitamins may aid in delaying or even reversing age-related neurodegeneration associated with cognitive decline among the elderly, as well as dementia and Alzheimer's disease [60]. Vitamins B6 and B12 participate as co-factors in homocysteine methylation and are associated with increased homocysteine concentrations. Possible roles of homocysteine in cognitive decline and dementia include: promotion of apoptosis, platelet activation, neurotoxicity arising from N-methyl-D-aspartate receptor activation and vascular injury associated with atherogenesis stimulation and smooth muscle cell proliferation [61]. Despite the suggested association of low serum concentrations of vitamins B6 and B12 with dementia and impaired cognition, to date there is no concrete evidence to suggest that supplementation prevents either of these conditions [62]. There is a need for well-designed large-scale trials to confirm the role of $\mathrm{B}$ vitamins and a potential benefit of their supplementation in these degenerative conditions.

\section{$B$ vitamins, neuropathy and neuropathic pain}

In addition to their important role in the maintenance of body functions, including repair, development, and growth, there is increasing evidence of an involvement of the B complex vitamins in the peripheral nervous system, in promoting acceleration of nerve repair, both in enhancement of nerve regeneration and recovery of nerve function $[3,4]$. Of particular note is the finding that experimental peripheral nerve injury leads to significant variations in nerve tissue levels of the B vitamins especially in the acute phase of injury, leading to the supplementation with $B$ complex vitamins in the acute period of peripheral nerve injury may be beneficial for accelerating nerve regeneration [3].

The B vitamins also play a role in nociception and may be employed for relief of pain and hyperalgesia. In addition to the neurological impact of B vitamin deficiencies, vitamins B1, B6, and B12 have been shown to exert anti-inflammatory and analgesic effects in animal models of chemical, electrical and thermal stimulation-induced pain. In clinical evaluations, the B-vitamins have been successfully used to address painful conditions such as carpal tunnel syndrome, lumbago, and neuropathies [42,63-70].

The suggested mechanisms of action by which vitamin B1 may exert an effect in pain relief include a blockade of metabolic damage pathways, including the advanced glycation end-product formation pathway, the diacylglycerol protein kinase $\mathrm{C}$ pathway, and the hexamine pathway $[65,71,72]$. Vitamin B1 also plays a role in modulation of neural excitability and $\mathrm{Na}+$ currents in injured DRG neurons, with a partial reversal of injury-induced density alterations and inactivation properties of tetrodotoxin-resistant and tetrodotoxinsensitive $\mathrm{Na}+$ currents and ramp currents in small DRG neurons [73]. Thiamine has also been suggested to mediate pain via its roles in trans ketolase activation (providing a shift for glucose to the pentose phosphate shunt) [74]; protein kinase B/Akt-mediated potentiation of angiogenesis and inhibition of apoptosis [75]; as well as antagonism of cerebral oxidative stress [76].

The roles of Vitamin B6 in pain relief and suppression of hyperalgesia include the participation in presynaptic inhibition of 
transmitter release from nociceptive afferent fibers carrying excitatory input to the spinal dorsal horn and thalamic neurons [77] as well as suppression of neuronal hyper-excitability [78]. Vitamin B6 has also been shown to to enhance inhibitory synaptic control by opioid or non-opioid mechanisms [79]. Additionally, vitamin B6 has been associated with an increase in brain levels of 5-hydroxytyptamine as an inhibitory transmitter, enhancing the inhibitory functions in central pain transmission [80].

The proposed mechanisms for a role of vitamin B12 in pain relief have included the promotion of nerve regeneration and/or remyelination by accumulation of exogenous B12 [67]. Because vitamin B12 may act as a methyl donor in DNA metabolism, high concentrations upregulate gene transcription, increasing protein synthesis for nerve regeneration [81]. Vitamin B12 may also be involved in selective blockade of sensory nerve conduction as a mechanism of action for vitamin B12 in painful conditions [82].

\section{Conclusion}

This updated review has briefly covered the physiological and biochemical properties of vitamins B1, B6 and B12. There is ample evidence for a role of these B vitamins in the nervous system, where they are active in metabolic and physiologic processes needed for maintenance and repair, in addition to an evident role in nerve repair regeneration, as evidenced by both deficiency states and studies in which they have been employed for analgesia and inflammation relief. Several mechanisms of action have been proposed for the role of these vitamins in the nervous system, and while further studies will undoubtedly shed more light on the specific mechanisms by which they exert their protective and therapeutic effects on the nervous system, it is currently clear that they do have an essential role. It is important to remember that as water-soluble vitamins with relatively little storage in the human body, maintenance of ideal levels through food and supplementation are vital. It is also important to note that RDAs are general guidelines, based broadly on the assumption that the populations of developed countries have access to proper nutrition and are not particularly at risk for deficiencies. There is however a wide spectrum of factors that can influence vitamin absorption and metabolism that must also be taken into account on a more individual basis, including diseases and medical/surgical history, lifestyle, age, and specific genetic polymorphisms, among others [1]. Furthermore, there is evidence that a large part of the population of the US and European nations does not reach RDA for many vitamins [83]. Consequently, there is a significant population with the potential to benefit from supplementation.

\section{References}

1. Kennedy DO (2016) B vitamins and the brain: mechanisms, dose and efficacy - a review. Nutrients 8: 68 .

2. Said HM (2011) Intestinal absorption of water-soluble vitamins in health and disease. Biochem J 437: 357-372.

3. Altun I, Kurutas EB (2016) Vitamin B complex and vitamin B12 levels after peripheral nerve injury. Neural Regen Res 11: 842-845.

4. Maladkar M, Tekchandani C, Dave U (2014) Post-marketing surveillance of fixed dose combination of methylcobalamine, alpha lipoic acid, folic acid, biotin, benfotiamine \& vitamin B6-nutripathy for the management of peripheral neuropathy. J Diabetes Mellitus 4: 124-132.

5. Merskey H, Bogduk N (1994) Part III: Pain Terms, A Current List with Definitions and Notes on Usage Classification of Chronic Pain, Second Edition, IASP Task Force on Taxonomy, edited by IASP Press, Seattle, PP: 209-214.
6. Treede RD, Jensen TS, Campbell JN, Cruccu G, Dostrovsky JO, et al. (2008) Redefinition of neuropathic pain and a grading system for clinical use: consensus statement on clinical and research diagnostic criteria. Neurology 70: 1630-1635.

7. Baron R, Binder A, Wasner G (2010) Neuropathic pain: diagnosis, pathophysiological mechanisms, and treatment. The Lancet Neurology 9: 807-819.

8. Baron R (2009) Neuropathic pain: a clinical perspective. Handb Exp Pharmacol 194: 3-30.

9. Basbaum AI, Bautista DM, Scherrer G, Julius D (2009) Cellular and molecular mechanisms of pain. Cell 139: 267-284.

10. Freynhagen R, Baron R (2009) The evaluation of neuropathic components in low back pain. Curr Pain Headache Rep 13: 185-190.

11. Otten JJ, Pitzi Hellwing J, Meyers LD (2009) Dietary Reference Intakes: The Essential Guide to Nutrient Requirements. National Academies Press, Washington, DC.

12. Martin P, Singleton CK, Hiller-Sturmhöfel S (2003) The role of thiamine deficiency in alcoholic brain disease. Alcohol Res Health 27: 174-181.

13. Butterworth RF, Shike M, Ross AC, Williams L, Baltimore W (2006) Modern Nutrition in Health and Disease, 10th Ed. USA, pp: 2027

14. Bâ A (2008) Metabolic and structural role of thiamine in nervous tissues. Cell Mol Neurobiol 23: 923-931.

15. Gubler CJ (1984) In: Machlin LJ, Dekker M (eds.) Handbook of vitamins: Nutritional, biochemical, and clinical aspects. New York, pp: 245.

16. Tallaksen CM, Tauboll E (2000) Excitatory effect of thiamine on CA1 pyramidal neurones in rat hippocampal slices in vitro. Eur J Neurol 7: 693-698.

17. Houzen H, Kanno M (1998) Thiamine and its derivatives inhibit delay rectifier potassium channels of rat cultured cortical neurons. Pharmacology 37: 313-322.

18. Goldberg DJ, Begenisich TB, Cooper JR (1975) Effects of thiamine antagonists on nerve conduction. II. Voltage clamp experiments with antimetabolites. J Neurobiol 6: 453-462.

19. Drewe J, Delco F, Kissel T, Beglinger C (2003) Effect of intravenous infusions of thiamine on the disposition kinetics of thiamine and its pyrophosphate. J Clin Pharm Ther 28: 47-51.

20. Sechi G, Serra A (2007) Wernicke's encephalopathy: new clinical settings and recent advances in diagnosis and management. Lancet Neurol 31: $442-455$.

21. Oliveira FA, Galan DT, Ribeiro AM, Cruz JS (2007) Brain Res 1134: 79-86.

22. Frank LL (2015) Thiamin in clinical practice. J Parenter Enteral Nutr 39: 503-520.

23. Kopelman MD, Thomson AD, Guerrini I (2009) The Korsakoff syndrome: clinical aspects, psychology and treatment. Alcohol Alcohol 44: $148-154$.

24. Scalzo SJ, Bowden SC, Ambrose ML, Whelan G, Cook MJ, et al. (2015) Wernicke-Korsakoff syndrome not related to alcohol use: a systematic review. J Neurol Neurosurg Psychiatry 86: 1362-1368.

25. Sechi G, Sechi E, Fois C, Kumar N (2016) Advances in clinical determinants and neurological manifestations of B vitamin deficiency in adults. Nutr Rev 74: 281-300.

26. Hellmann H, Mooney S (2010) Vitamin B6: A molecule for human health? Molecules 15: 442-459.

27. Mooney S, Leuendorf JE, Hendrickson C (2009) Vitamin B6: A long known compound of surprising complexity. Molecules 14: 329-351.

28. Drewke C, Leistner E (2001) Biosynthesis of vitamin B6 and structurally related derivatives. Vitam Horm 61: 121-155.

29. MittenHuber G (2001) Phylogenetic analyses and comparative genomics of vitamin B6 (pyridoxine) and pyridoxal phosphate biosynthesis pathways. J Mol Microbiol Biotechnol 3: 1-20.

30. Nakamura MT, Nara TY (2004) Structure, function, and dietary regulation of delta-6, delta-5, and delta-9 desaturases. Ann Rev Nutr 24: 345-367. 
31. Cheltsov AV, Guida WC, Ferreira GC (2003) Circular permuation of 5aminolevulinate synthase: effect on folding, conformational stability, and structure. J Biol Chem 278: 27945-27955.

32. Coombs GF (2012) The vitamins: Fundamental aspects in nutrition and health. 4th edn San Diego, Academic Press, p: 570.

33. Wondrak GT, Roberts MJ, Jacobson MK (2004) 3-hydroxypyridine chromophores are endogenous sensitizers of photooxidative stress in human skin cells. J Biol Chem 279: 30009-30020.

34. Müller IB, Wu F, Bergmann B, Knöckel J, Walter RD, et al. (2009) Poisoning pyridoxal 5-phosphate-dependent enzymes: a new strategy to target the malaria parasite Plasmodium falciparum. PLoS One 4: 4406.

35. Percudani R, Peracchi A (2003) A genomic overview of pyridoxalphosphate-dependent enzymes. EMBO Rep 4: 850-854.

36. Ehrenshaft M, Bilski P, Li MY, Chignell CF, Daub ME (1999) A highly conserved sequence is a novel gene involved in de novo vitamin B6 biosynthesis. Proc Natl Acad Sci USA 96: 9374-9378.

37. Kannan K, Jain SK (2004) Effect of vitamin B6 on oxygen radicals, mitochondrial membrane potential, and lipid peroxidation in $\mathrm{H} 202$-treat U937 monocytes. Free Radic Biol Med 36: 423-428.

38. Hsu CC, Cheng CH, Hsu CL, Lee WJ, Huang SC (2015) Role of vitamin B6 status on antioxidant defenses, glutathione, and related enzyme activities in mice with homocysteine-induced oxidative stress. Food Nutr Res 59: 25702.

39. Kumar N (2010) Neurologic presentations of nutritional deficiencies. Neurol Clin 28: 107-170.

40. Leklem JE, Machlin LJ Dekker M (1961) Vitamin B6. In: Handbook of vitamins New York.

41. Hammond N, Wang Y, Dimachkie M, Barohn R (2013) Nutritional Neuropathies. Neurol Clin 31: 477-489.

42. Aufiero E, Stitk TP, Foye PM, Chen B (2004) Pyridoxine hydrochloride treatment of carpal tunnel syndrome: a review. Nutr Rev 62: 96-104.

43. Brown KL (2005) Chemistry and Enzymology of Vitamin B12. Chem Rev 105: 2075-2149.

44. Kräutler B (2005) Vitamin B12: chemistry and biochemistry. Biochem Soc Trans 33: 806-810.

45. Matthews RG (2009) Cobalamin- and Corrinoid-Dependent Enzymes. In Metal Ions in Life Sciences; Sigel A, Sigel H, Sigel RKO, (eds) Royal Society of Chemistry: Cambridge, UK 6: 53.

46. Nishimoto S, Tanaka H, Okamoto M, Okada K, Murase T (2015) Methylcobalamin promotes the differentiation of Schwann cells and remyelination in lysophosphatidylcholine-induced demyelination of the rat sciatic nerve. Front Cell Neurosci 9: 298.

47. Randaccio L, Geremia S, Demitri N (2010) Vitamin B12: Unique metalorganic compounds and the most complex vitamins. Molecules 15: 3228-3259.

48. Zingg JM, Jones PA (1997) Genetic and epigenetic aspects of DNA methylation on genomic expression, evolution, mutation, and carcinogenesis. Carcinogenesis 18: 869-882.

49. Carmel R (2003) Mild transcobalamin I (haptocorrin) deficiency and low serum cobalamin concentrations. Clin Chem 49: 1367-1374.

50. McEvoy GK (2000) American Hospital Formulary Service - Drug Information 2000. Bethesda, MD: American Society of Health-System Pharmacists, (Plus Supplements) pp 3319-3325.

51. Chawla J, Kvarnberg D (2014) Hydrosoluble vitamins. Handb Clin Neurol 120: 915-926.

52. Poli D, Antonucci E, Cecchi E, Marcucci R, Liotta AA (2005) Culprit factors for the failure of well conducted warfarin therapy to prevent ischemic events in patients with atrial fibrillation. The role of homocysteine. Stroke 36: 2159-2163.

53. Halsted CH, Dekker M (1995) Alcohol and folate interactions: clinical implications. In: Bailey (eds.) Folate in Health and disease, New York, pp: 313-335.

54. Gropper SS, Smith JL (2013) Water-soluble vitamins. In: Gropper SS (eds.) Advanced nutrition and human metabolism, Belmont, CA, Wadsworth, pp: 307-366.
55. Dubel SJ, Soloff LA (1963) The effect of thiazides on thiamine excretion and on pyridoxine metabolism. Am J Med Sci 245: 92-96.

56. Cook CCH, Hallwood PM, Thomson AD (1998) B vitamin deficiency and neuropsychiatric syndromes in alcohol misuse. Alcohol Alcohol 33: 317-336.

57. Shane B (2008) Folate and vitamin B12 metabolism: overview and interaction with riboflavin, vitamin B6, and polymorphisms. Food Nutr Bull 29: S5-S16.

58. McCormick DD (1989) Two interconnected B vitamins: riboflavin and pyridoxine. Physiol Rev 69: 1170-1198.

59. Morris MS, Selhub J, Jacques PF (2012) Vitamin B-12 and folate status in relation to decline in scores on the Mini-Mental State Examination in the Framingham Heart Study. J Am Geriatr Soc 60: 1457-1464.

60. Gillette Guyonnet S, Abellan Van Kan G, Andrieu S, Barberger Gateau P, Berr C (2007) IANA task force on nutrition and cognitive decline with aging. J Nutr Health Aging 11: 132-152

61. Garcia A, Zanibbi K (2004) Homocysteine and cognitive function in elderly people. CMAJ 171: 897-904.

62. Gillette Guyonnet S, Secher M, Vellas B (2013) Nutrition and neurodegeneration: epidemiological evidence and challenges for future research. BJCP 75: 738-755.

63. Devathasan G, Teo WL, Mylvaganam A (1986) Methylcobalamin in chronic diabetic neuropathy. A double-blind clinical and electrophysiological study. Clin Trials J 23: 130-140.

64. Gokhale LB (1996) Curative treatment of primary spastic dysmenorrhea. Indian J Med Res 103: 227-231.

65. Haupt E, Ledermann H, Köpcke W (2005) Benfotiamine in the treatment of diabetic polyneuropathy - a three-week randomized, controlled pilot study (BEDIP study). Int J Clin Pharmacol Ther 43: 71-77.

66. Kuwabara S, Nakazawa R, Azuma N, Suzuki M, Miyajima K (1999) Intravenous methylcobalamin treatment for uremic and diabetic neuropathy in chronic hemodialysis patients. Internal Medicine 38: 472-475.

67. Mauro GL, Martorana U, Cataldo P, Brancato G, Letizia G (2000) Vitamin B12 in low back pain: a randomised, double-blind, placebo-controlled study. Eur Rev Med Pharmacol Sci 4: 53-58.

68. Várkonyi T, Kempler P (2008) Diabetic neuropathy: new strategies for treatment. Diabetes, Obesity and Metabolism 10: 99-108.

69. Woelk H, Lehrl S, Bitsch R (1998) Benfotiamine in treatment of alcoholic polyneuropathy: an 8-week randomized controlled study (BAP I Study). Alcohol \& Alcoholism 33: 631-638.

70. Yaqub BA, Siddique A, Sulimani R (1992) Effects of methylcobalamin on diabetic neuropathy. Clin Neurol Neurosurg 94: 105-111.

71. Sánchez-Ramírez GM, Caram-Salas NL, Rocha-González HI, VidalCantú GC, Medina-Santillán R (2006) Benfotiamine relieves inflammatory and neuropathic pain in rats. Eur J Pharmacol 530: 48-53.

72. Winkler G, Pál B, Nagybégányi E, Öry I, Porochnavec M (1999) Effectiveness of different benfotiamine dosage regimens in the treatment of painful diabetic neuropathy. Arzneimittelforschung 49: 220-224.

73. Song XS, Huang ZJ, Song XJ (2009) Thiamine suppresses thermal hyperalgesia, inhibits hyperexcitability, and lessens alterations of sodium currents in injured, dorsal root ganglion neurons in rats. Anesthesiology 110: 387-400.

74. Brownlee M, Banting Lecture (2005) The pathobiology of diabetic complications. Diabetes 54: 1615-1625.

75. Gadau S, Emanueli C, Van Linthout S, Graiani G, Todaro M, et al. (2006) Benfotiamine accelerates the healing of ischaemic diabetic limbs in mice through protein kinase B/Akt-mediated potentiation of angiogenesis and inhibition of apoptosis. Diabetologia 49: 405-420.

76. Wu S, Ren J (2006) Benfotiamine alleviates diabetes-induced cerebral oxidative damage independent of advanced glycation end-product, tissue factor and TNF-alpha. Neurosci Lett 394: 158-162.

77. Schmidt RF (1971) Presynaptic inhibition in the vertebrate central nervous system. Rev Physiol Biochem Exp Pharmacol 63: 20-101 
Citation: Geller M, Oliveira L, Nigri R, Mezitis SG, Ribeiro MG, et al. (2017) B Vitamins for Neuropathy and Neuropathic Pain. Vitam Miner 6: 161.

Page 7 of 7

78. Matsuda M, Hori S, Asakura T, Kurioka S (1987) Effects of carbon dioxide on onsets of seizures in mice induced by antagonists of vitamin B6. Biochem Pharmacol 36: 2040-2042.

79. Willis W (1982) Control of nociceptive transmission in the spinal cord. In: Progress in Sensory Physiology, pp: 1-159.

80. Zimmermann M, Bartoszyk GD, Bonke D, Jurna I, Wild A (1990) Antinociceptive properties of pyridoxine. Ann NY Acad Sci 585: 219-230.

81. Pfohl-Leszkowicz A, Keith G, Dirheimer G (1991) Effect of cobalamin derivatives on in vitro enzymatic DNA methylation: methylcobalamin can act as a methyl donor. Biochemistry 30: 8045-8051.
82. Takeshige C, Ando Y, Ando M (1971) Effects of vitamin B12 and aldosterone on the conduction of sensory and motor nerve impulse. Vitamins 44: 272- 282.

83. Troesch B, Hoeft B, McBurney M, Eggersdorfer M, Weber P (2012) Dietary surveys indicate vitamin intakes below recommendations are common in representative Western countries. Br J Nutr 108: 692-698. 